\title{
Investigating the mechanism of acoustically activated uptake of drugs from Pluronic micelles Ghaleb A Husseini ${ }^{1}$, Christopher M Runyan ${ }^{2}$ and William G Pitt*1
}

Address: ${ }^{1}$ Department of Chemical Engineering, Brigham Young University, Provo, Utah, USA 84602, USA and ${ }^{2}$ Department of Microbiology, Brigham Young University, Provo, Utah 84602, USA

E-mail: Ghaleb A Husseini - ghaleb_husseini@hotmail.com; Christopher M Runyan -cmr22@email.byu.edu; William G Pitt* - pitt@byu.edu ${ }^{*}$ Corresponding author

Published: 30 August 2002

BMC Cancer 2002, 2:20
Received: 18 April 2002

Accepted: 30 August 2002

This article is available from: http://www.biomedcentral.com/I47/-2407/2/20

(C) 2002 Husseini et al; licensee BioMed Central Ltd. This article is published in Open Access: verbatim copying and redistribution of this article are permitted in all media for any non-commercial purpose, provided this notice is preserved along with the article's original URL.

\begin{abstract}
Background: This paper examines the mechanism of ultrasonic enhanced drug delivery from Pluronic micelles. In previous publications by our group, fluorescently labeled Pluronic was shown to penetrate HL-60 cells with and without the action of ultrasound, while drug uptake was increased with the application of ultrasound.
\end{abstract}

Methods: In this study, the amount of uptake of two fluorescent probes, Lysosensor Green (a pHsensitive probe) and Cell Tracker Orange CMTMR (a pH-independent probe), was measured in HL-60 and HeLa cells.

Results: The results of our experiments show that the increase in drug accumulation in the cells as a result of ultrasonication is not due to an increase in endocytosis due to ultrasonication.

Conclusions: We hypothesize that sonoporation plays an important role in the acoustically activated drug delivery of chemotherapy drugs delivered from Pluronic micelles.

\section{Background}

In order to overcome the unwanted effects associated with conventional chemotherapy, the use of molecular vehicles to sequester the therapeutic drug in a package and release it to the tumor site at the appropriate time is a topic of vigorous investigation. Such a vehicle would protect healthy cells in the host body from interaction with the chemotherapeutic agents and therefore would help to alleviate unwanted side effects. The main challenge is to completely sequester the drug, but yet be able to release it upon demand in both time and space. Ultrasound is an ideal mediator of drug delivery because it provides both temporal and spatial control over irradiation of tissue and subsequent delivery of the drug.

Results of the comet (DNA degradation) assay presented in a previous publication [1] show that Doxorubicin
(Dox) released from Pluronic micelles eventually binds to the DNA and causes it to fragment. In another publication, our group reported that Dox and Ruboxyl (Rb) are released from the core of Pluronic P105 micelles upon exposure to ultrasound at frequencies below $100 \mathrm{kHz}$ [2]. Ultrasonic release of Dox and $\mathrm{Rb}$ from these micelles is evident. However, the question remains as to if and how ultrasound enhances uptake of Dox by the cell. This paper probes and discusses possible mechanisms by which the P105 micelles and the ultrasound drug delivery system induce drug uptake by cancer cells. Three postulated mechanisms are presented and discussed below. They are 1) ultrasound releases drug from the micelle and also perturbs the cell membrane which increases the permeability of the cells toward the drug and Pluronic; 2) ultrasound releases drug from micelles and the drug is taken into the cells by normal passive modes; 3 ) ultrasound induces an 
increase in the active endocytosis of the micelles (with drug) into the cell.

\section{Methods \\ Cells}

HL-60 cells were cultured in Roswell Park Memorial Institute medium, RPMI-1640 (HyClone, Logan, UT, USA), supplemented with $10 \%$ fetal calf serum, $6 \mathrm{mM} \mathrm{L-}$ glutamine and $7.5 \%$ sodium bicarbonate. The culture was maintained in $75-\mathrm{ml}$ plastic tissue flasks at $37^{\circ} \mathrm{C}$ in humidified $5 \% \mathrm{CO}_{2}$ and was passaged every 3 to 4 days.

HeLa cells were cultured in Minimum Essential Medium, $\alpha$ MEM (HyClone, Logan, UT, USA), supplemented with $10 \%$ fetal calf serum, L-glutamine, non-essential amino acids and sodium pyruvate. The culture was also maintained in $75-\mathrm{ml}$ plastic tissue flasks at $37^{\circ} \mathrm{C}$ in humidified $5 \% \mathrm{CO}_{2}$ and was passaged every 2 to 3 days. HeLa cells have a high rate of non-receptor mediated endocytosis [3].

\section{Fluorescent probes}

In order to test the hypothesis that endocytosis is involved in micelle uptake, a probe that fluoresces more strongly in acidic environments was used, namely Lysosensor Green (Molecular Probes, L-7535, Eugene, OR). Lysosensor Green has a pKa of 5.2, which gives it a very low fluorescence until it enters inside acidic compartments of the cell. The $\mathrm{pH}$ inside endosomes is about 4.8 , and therefore this probe will show an increase in fluorescence when it is located in the endosome.

As a control in some experiments, a non-pH sensitive fluorescent probe was used, namely Cell Tracker Orange CMTMR (C-2927, Molecular Probes, Eugene, OR).

\section{Measuring the partition coefficient}

Lysosensor Green is hydrophobic, which indicates that in aqueous Pluronic solutions it will partition to the poly (propylene oxide) core of the Pluronic micelles, similar to the manner in which Doxorubicin and Ruboxyl partition to the core [4]. The partition coefficient of Lysosensor Green between phosphate buffered saline (PBS) $(\mathrm{pH}=$ 7.4 ) and poly (propylene oxide) (PPO, MW $=4500 \mathrm{~g} / \mathrm{mol}$, Sigma Chemicals) was determined using the fluorescent characteristics of the probe. Solutions with a known concentration of Lysosensor Green in both PBS and PPO were prepared and placed in a fluorometer. The $\mathrm{pH}$ was kept at 7.4. The samples were excited at $443 \mathrm{~nm}$, and the emission at $505 \mathrm{~nm}$ was measured. From these data, a calibration plot of probe concentration vs. emission intensity was prepared in both PPO and PBS.

Next, Lysosensor Green (final concentration $1 \mu \mathrm{M}$ ) was placed into a vial containing both PBS and PPO and was magnetically stirred overnight in order to allow equilibrium to be established. This produced an emulsion that was broken by centrifugation. The water and PPO phases were separated, and the emission intensity was measured for each sample. The concentration of the probe in each phase was determined from calibration plots, from which the partition coefficient was calculated.

\section{Flow cytometry}

One ml of HL-60 cells $\left(1 \times 10^{6}\right)$ was mixed with $1 \mathrm{ml}$ of solutions of a fluorescent probe (final concentration of the probe was about $1 \mu \mathrm{M}$ ). After one hour of incubation or ultrasonication, the cell fluorescence was determined using a flow cytometer (Coulter EPICS-XL cytometer, Coulter Corp., Hialeah, FL), and the fluorescence was compared between the ultrasonicated and non-ultrasonicated cells.

\section{Ultrasonication}

Ultrasound power was generated by a Sonicor SC-100 ultrasonicating bath (Sonicor Instr., Copaique, N. Y.) operating at $70 \mathrm{kHz}$. The power density was controlled by adjusting the input voltage using a variable A.C. transformer (variac). The insonation intensity as a function of applied voltage was determined using a calibrated hydrophone (Bruel and Kjaer model 8103, Decatur, GA). The acoustic intensity generated by the Sonicor bath increased with applied voltage. Following calibration experiments, the variac voltage was adjusted to produce an ultrasonic intensity of $1.2 \mathrm{~W} / \mathrm{cm}^{2}$. Since we were not studying the effect of hyperthermia induced by ultrasonication, the temperature of the bath was maintained at $37^{\circ} \mathrm{C}$ using a recirculating thermostatic bath.

\section{Results}

The partition coefficient of the Lysosensor Green between the PPO and PBS phases was measured to be 13; i.e., for every 13 molecules present in the PPO, one molecule is present in the surrounding aqueous solution. The measurement of the partition coefficient was not completely accurate, because some of the PPO molecules were still present in the water phase even after the sample was centrifuged three times. This causes the amount of the probe measured in the water phase to be larger than if the water were pure, indicating that the true partition coefficient is larger than 13. The high value of the partition coefficient indicates that the Lysosensor Green probe is fairly hydrophobic, which suggests that it will accumulate inside the poly (propylene oxide) core of the P105 micelle, similarly to the accumulation of Dox and other hydrophobic drugs.

Flow cytometry was used to determine if the mode of drug uptake was related to endocytosis. To do so, two cell lines were used, HL-60 cells with a relatively low amount of en- 


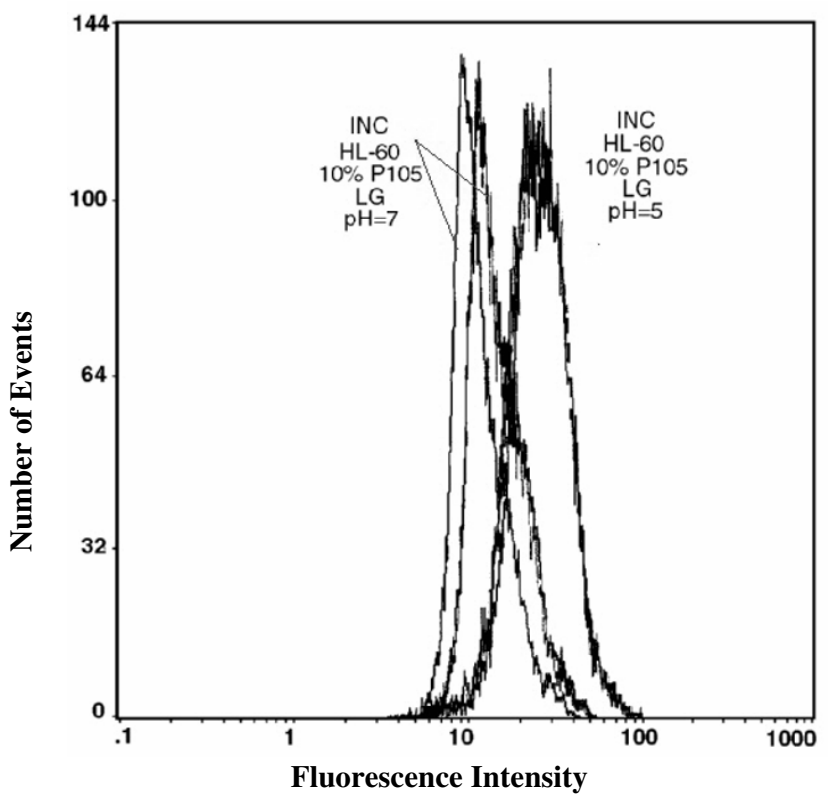

\section{Figure I}

A histogram of fluorescence events vs. fluorescent intensity for HL-60 cells. When cells were incubated at $\mathrm{pH} 5.0$ encapsulated in 10\% PI05 (right side histograms), the fluorescence of Lysosensor Green in the cells was greater than that at $\mathrm{pH}$ 7.0 in the presence of 10\% PI05 (left side histograms).

docytotic activity, and HeLa cells which have a relatively high amount of non-receptor mediated endocytosis [3].

Figure 1 shows a positive control, in which the $\mathrm{pH}$ of the HL-60 cells exposed to Lysosome Green encapsulated in 10\% P105 was reduced to 5.0 and compared with cells incubated in the presence of 10\% P105 at neutral pH. The xaxis shows the amount of fluorescence emitted from the cells and the $y$-axis is the number of cells that exhibited the amount of fluorescence shown on the x-axis. At the lower pH, HL-60 cell fluorescence increased substantially, indicating that the probe associated with the acidified cells is in an acidic environment; therefore the probe appears to be responsive to low $\mathrm{pH}$ such as found inside a low-pH lysosome.

HeLa cells were also used as another positive control. At normal $\mathrm{pH}(7.1-7.4)$ the $\mathrm{pH}$-sensitive probe encapsulated in 10\% P105 showed stronger fluorescence in the HeLa cells than the non $\mathrm{pH}$ sensitive probe in the presence of 10 $\%$ P105, indicating that HeLa cells display strong endocytotic activity and incorporate the probe into acidic vesicles (Figure 2).

Figure 3 shows the amount of fluorescence for negative control HL-60 cells (left), cells incubated with Lysosensor

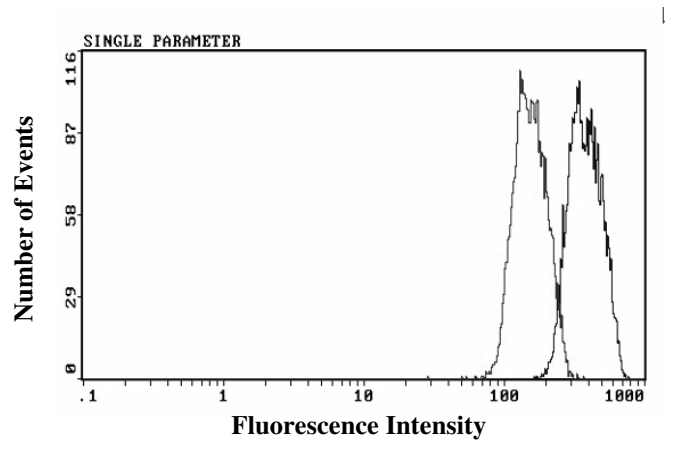

Figure 2

A histogram of emissions from HeLa cells incubated with $\mathrm{pH}$ sensitive probe and 10\% PI05 (right) and the $\mathrm{pH}$ independent probe encapsulated in PI05 (left).

Green encapsulated in 10\% P105 (middle), and cells incubated with Lysosensor Green in PBS (right). As was found previously for Dox [1], less Lysosensor Green is associated with the cells when the hydrophobic probe is encapsulated in the Pluronic micelle; much less encapsulated probe was able to accumulate in low-pH compartments in the cells than free probe introduced from a solution of PBS. This is consistent with our conviction that hydrophobic molecules partition to the micellar core and are not taken up as readily by cells.

Figure 4 shows that ultrasound produces no difference in Lysosensor Green fluorescence in HL-60 cells when the probe is encapsulated in P105 micelles. The cells were either incubated or ultrasonicated for 1 hour in the presence of Lysosensor Green in 10\% P105. For each condition, three replicates are shown in the figure. The observation that the incubated and ultrasonicated cell histograms overlap closely (see Figure 4) suggests that the ultrasound does not cause the probe to partition to a more acidic environment anymore than it does without ultrasound.

Figures 4 and 5 show the results with the HL-60 as a result of incubating and sonicating these cells with the $\mathrm{pH}$ sensitive probe (Figure 4) and the non-pH sensitive probe (Figure 5). Figure 5 shows a slight increase in the amount of fluorescence as a result of ultrasonication, while Figure 4 shows no increase in the amount of fluorescence as a result of ultrasonication. These data indicate that ultrasound enhances the uptake of a fluorescent probe into the cells as shown with the non-pH sensitive probe in Figure 5 , but does not enhance the amount of the probe present in a low-pH compartment as shown with the $\mathrm{pH}$-sensitive probe in Figure 4. This suggests that the ultrasound enhances the uptake of hydrophobic molecules, but the en- 


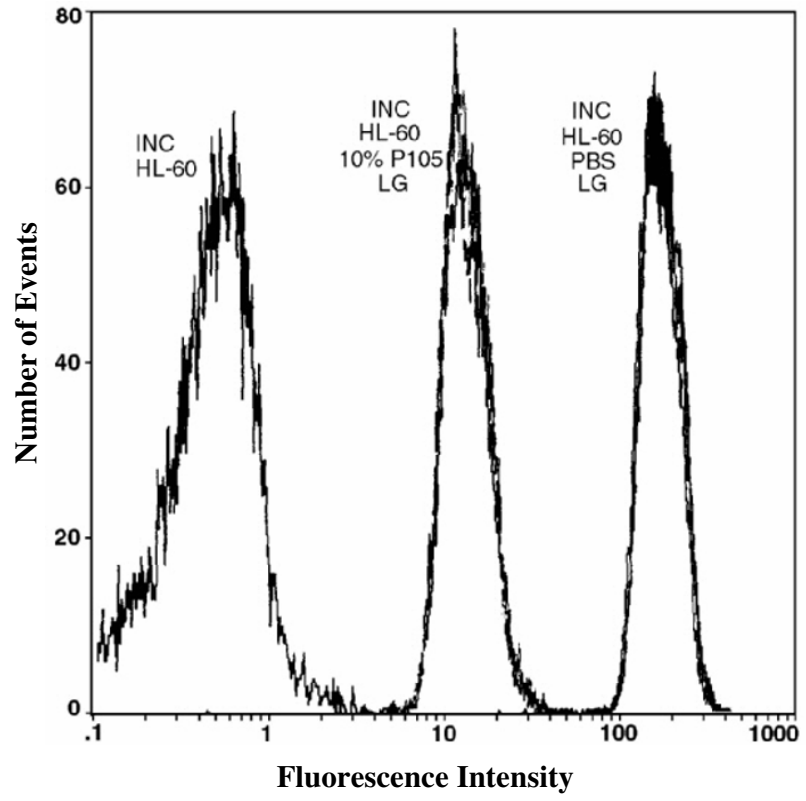

Figure 3

A histogram of fluorescent events vs. fluorescent intensity for HL-60 cells. Left: control cells with no fluorescent probe added. Center: cells that have been incubated with the fluorescent probe in the presence of 10\% PI05 micelles for one hour. Right: cells that have been incubated with the fluorescent probe in a solution of PBS for one hour. Using Lysosensor Green and flow cytometry, more fluorescence is observed when the probe is introduced in a solution of PBS than when encapsulated in PI05.

hanced uptake does not appear to involve low-pH compartments.

\section{Discussion and conclusions}

The results of these and of previously reported experiments allow evaluation of several critical hypotheses regarding the mechanisms of this ultrasonically enhanced drug delivery system. We have previously established the fact that ultrasound releases Dox and other drugs from micelles [2,5-10]. The following discussion pertains to if and how ultrasound also enhances uptake of the drug into the cells. We will consider both passive transport via diffusion through the cell membrane, and active transport in the cell via endocytosis.

\section{Hypothesis \# I}

Ultrasound perturbs the cell membrane and/or cell wall to render it more permeable to the drug. Ultrasound creates stress on cells by at least two mechanisms. The first is acoustic streaming in which momentum from directed propagating sound waves is transferred to the liquid, causing the liquid to flow in the direction of the sound prop-

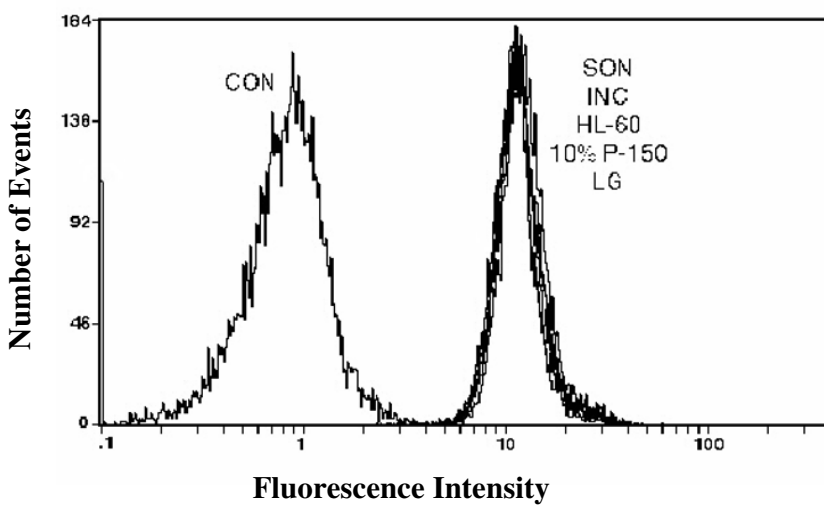

Figure 4

A histogram showing that no difference in fluorescence is observed between HL-60 cells incubated and ultrasonicated for one hour (at I W/cm ${ }^{2}$ and $70 \mathrm{kHz}$ ) in the presence of 10\% PI05 and Lysosensor Green (histograms with incubated cells overlap with histograms of ultrasonicated cells). The histogram on the left is a control with no fluorescent probe added. The 6 histograms on the right are incubated and ultrasonicated HL-60 cells (3 replicates each) in the presence of $10 \%$ PI05.

agation [11]. Acoustic streaming happens to varying degrees at any intensity of ultrasound. The second mechanism is microconvection created by oscillating gas bubbles in the liquid. The cycles of low and high acoustic pressure cause the micron-sized gas bubbles to expand and shrink, which in turn creates shear flow around the oscillating bubbles. Stable cavitation results when the acoustic intensity is sufficiently low that the bubbles do not collapse completely during their contraction cycle. Collapse or transient cavitation is produced when the bubble collapses during the contraction cycle [12]. Both stable and collapse cavitation create high shear stresses in the fluid which can damage the cell membrane. The sudden collapse produces a shock wave, and the adiabatic compression of the gas produces temperatures on the order of $5000 \mathrm{~K}$, which in turn fragments water and other molecules into free radicals [12]. The general phenomenon of creating holes or pores in cell membranes via these intense acoustic forces is called sonoporation.

Tachibana et al. have shown that the exposure of HL-60 cells to $255 \mathrm{kHz}$ of ultrasound and merocyanine 540 (a photosensitive durg) for 30 seconds formed pores in the cell membrane [13]. Electron micrographs showed that the cytoplasm of some cells seemed to have extruded through the pores formed in the cell membrane as a result of sonoporation. When cells were exposed to ultrasound alone, the cell membrane showed minor disruptions. 


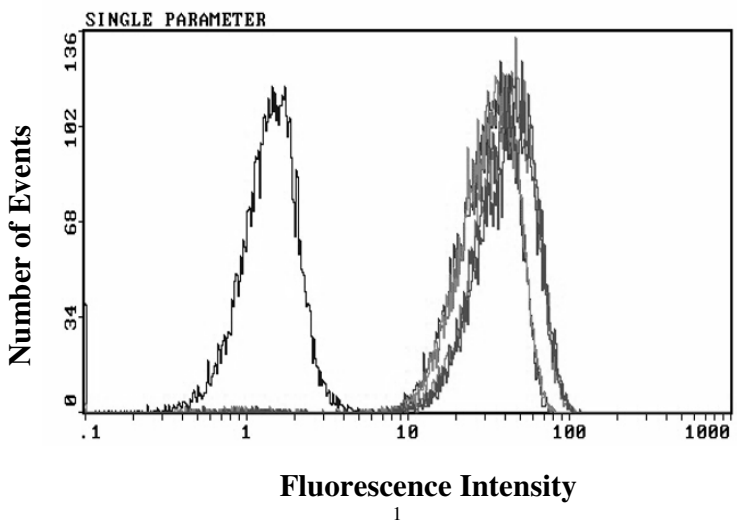

\section{Figure 5}

A histogram of HL-60 cells incubated (middle, 3 replicates) and ultrasonicated (right, 3 replicates) with the non-pH sensitive probe Cell Tracker Orange CMTMR. The histogram on the left represents the control with no fluorescent probes added.

Saito et al. showed that exposure to ultrasound increased the permeability of corneal endothelium cells [14]. The increase in permeability appeared to be reversible and the cells regained their membrane integrity after several minutes.

The experiments reported herein using flow cytometry have shown that more of the non-pH sensitive probe (C2927) accumulates in HL-60 cells during the application of ultrasound (Figure 5). This is consistent with our previous results showing that more Dox and Ruboxyl accumulate in the cells during ultrasonic exposure $[1,6,10,15]$. We postulate that the shear events associated with ultrasound and accompanying cavitation events leads to stress on the cell membrane and/or the cell wall and renders the cell more permeable to the passive transport of the drugs or the fluorescent probes. In addition to rendering the membrane more permeable, ultrasound simultaneously releases the drug (or other molecules) from the micelles, thus increasing the external local concentration. None of our data nor of literature reports leads us to reject hypothesis \#1, and in fact supports it. Hence, ultrasound of the intensity and frequency used herein appears to make cell membranes more permeable.

\section{Hypothesis \#2}

Ultrasound only increases the external drug concentration, but does not change the permeability of the cell membrane. If this alternative hypothesis were true, the increased drug concentration outside the cells (and increased driving force for diffusion) would lead to faster and greater drug uptake into the cells by the same passive routes as without ultrasound. It may also be argued that ultrasound can reduce the mass transport boundary layer around the cells (due to microconvection currents) and hence enhance the diffusion of the drug to the cell membrane. If this small mass transfer boundary layer were the limiting diffusive barrier to membrane transport, then the reduction in boundary layer thickness would indeed increase transport into the cell. However, transport into the cell is a serial process, and the external boundary layer is only one step, and for water-soluble molecules this resistance is probably much smaller than the resistance of transport across the lipid membrane of the cell. We posit that the controlling resistance is that of the cell membrane, and therefore ultrasonic reduction of the external mass transfer boundary, without any decrease in the cell membrane resistance would not measurably increase the transport into the cell. Thus we dismiss the possibility that solely the decrease in the mass transfer boundary layer surrounding the cell (due to insonation) would cause a significant increase in drug uptake.

It is true that ultrasound releases drug from the micelles, and thus the external concentration of free drug is increased. To test the second hypothesis we need another tracer besides Dox that crosses the cell membrane, a tracer that does not change its external concentration upon insonation. Rapoport showed that ultrasonication increased the amount of fluorescently labeled P-105 that entered the cell (along with increased Dox) [16]. Since the external P-105 concentration does not change upon insonation, the only way for ultrasound to increases its uptake is if the cell membrane were rendered more permeable to P105 transport. Thus ultrasound increases the membrane permeability, and we can reject hypothesis \#2 and accept hypothesis \#1.

\section{Hypothesis \#3}

Ultrasound enhances endocytosis or other activated uptake of the entire micelle (with the encapsulated drug) into the cancer cells. Previous research has established that endocytosis of drug-containing Pluronic micelles occurs [16]. However, the question remains as to whether ultrasound triggers an increase in the endocytotic process that transports micelles in the cells.

In order to test the postulate that the cells are taking the drug in by endocytosis, a probe that fluoresces more strongly in acidic environments was used, namely Lysosensor Green. During normal endocytosis, the great majority of endosomes fuse with a primary lysosome to form a secondary lysosome. The $\mathrm{pH}$ inside lysosomes is about 4.8 while the $\mathrm{pH}$ outside these compartments is around 7.1; therefore Lysosensor Green would show an increase in fluorescence when taken up in an endosome and transferred to a lysosome. 
The data in Figure 4 show that ultrasound does not increase the amount of Lysosensor Green uptake by the HL60 cells. Neither did it increase the uptake in HeLa cells. This data indicates that ultrasound enhances the uptake of the probe into the cells but does not enhance the amount of the probe present in a $\mathrm{pH}$ sensitive compartment. This suggests that the ultrasound enhances drug uptake, but not by stimulating an increase in the extent of endocytosis.

In summary, entrance of drug into the cells under normal conditions appears to involve endocytosis and acidic vesicles as well as perhaps some transport through the cell membrane into the cytoplasm. Application of ultrasound increases the rate of uptake by this latter route, but does not appear to increase the uptake of drug into acidic vesicles, which still occurs at the normal rate. Although ultrasound releases drug from the Pluronic micelles, the enhanced uptake appears to be due to more than increased passive diffusion due only to increased external concentration of free drug. Apparently this intensity of ultrasound perturbs the cell membrane, making it more permeable to drug transport, at least by released drug, and perhaps by whole micelles passing through perturbations in the membrane.

This is a very important observation for this drug delivery system, because it indicates that healthy cells that are not exposed to ultrasound when the drug is introduced from Pluronic micelles will not be affected as adversely by the micelle contents compared to tissues exposed to ultrasound. These data imply that P105 micelles effectively sequester other hydrophobic molecules in addition to anthracycline drugs.

\section{Competing interests}

None declared

\section{Authors' contributions}

Ghaleb Husseini was responsible for growing the cells, preparing the encapsulated micelles solution, measuring the partition coefficient, incubating and sonicating the cells and preparing the manuscript.

Chris Runyan was responsible for operating the flow cytometer and helping in the analysis of the data.

William Pitt helped in the design of the experiments, and in writing the manuscript.

\section{Acknowledgments}

The authors would like to acknowledge funding from the National Institute of Health (CA 76562) and (HL 59923).

\section{References}

I. Husseini GA, El-Fayoumi RI, O'Neill KL, Rapoport NY, Pitt WG: DNA Damage Induced by Micellar-Delivered Doxorubicin and Ultrasound: Comet Assay Study. Cancer Lett 2000, I 54:2 I I216

2. Husseini GA, Myrup GD, Pitt WG, Christensen DA, Rapoport NY: Factors Affecting Acoustically-Triggered Release of Drugs from Polymeric Micelles. Journal of Controlled Release 2000, 69:4352

3. Olson JK, Grose C: Endocytosis and recycling of varicellazoster virus Fc receptor glycoprotein gE: internalization mediated by a YXXL motif in the cytoplasmic tail. J Virol 1997, 7 I:4042-52

4. Rapoport NY, Herron JN, Pitt WG, Pitina L: Micellar delivery of doxorubicin and its paramagnetic analog, ruboxyl, to HL-60 cells: effect of micelle structure and ultrasound on the intracellular drug uptake. Journal of Controlled Release I999, 58: I 53-I62

5. Husseini GA, Pitt WG, Al-Fayoumi RY, O'Neill KL, Rapoport NY: Acoustically Activated Delivery of Doxorubicin to HL-60 Cells from Pluronic (P-I05) Micelles: A Single Cell Electrophoresis Assay (Comet Assay) Study. in AICHE 1999 Annual Meeting. Dallas, Texas, USA: American Institute of Chemical Engineers. 1999

6. Munshi N, Rapoport N, Pitt WG: Ultrasonic activated drug delivery from Pluronic P- 105 micelles. Cancer Letters 1997, I I 7: I -7

7. Rapoport N, Pitina L, Munshi N, Pitt WG: Acoustically Activated Localized Drug Delivery. in 8th International Symposium on Recent Advances in Drug Delivery Systems. Salt Lake City, Utah. 1997

8. Rapoport N, Munshi N, Pitina L, Pitt WG: Pluronic Micelles as Vehicles for Tumor-Specific Delivery of Two Anti-Cancer Drugs to HL-60 Cells Using Acoustic Activation. Polymer Preprints 1997, 38(2):620-621

9. Rapoport N, Munshi N, Pitt WG: Acoustically Activated Drug Delivery from Pluronic Micelles. in 3rd International Symposium on Polymer Therapeutics. London: University of London. 1998

10. Rapoport N, Pitina L: Intracellular distribution and intracellular dynamics of a spin-labeled analog of Doxorubicin by fluorescence and EPR spectroscopy. J Pharm Sci 1998, 87:321-325

II. Rooney JA: Other Nonlinear Acoustic Phenomena, in Ultrasound Its Chemical, Physical, and Biological Effects: (Edited by: S. Suslick) VCH: New York 1988, 65-96

12. Atchleyand AA, Crum LA: Acoustic Cavitation and Bubble Dynamics, in Ultrasound Its Chemical, Physical, and Biological Effects, (Edited by: KS Suslick) VCH Publishers: New York. 1988, I-64

13. Tachibana K, Uchida T, Ogawa K, Yamashita N, Tamura K: Induction of cell-membrane porosity by ultrasound. Lancet 1999, 353:1409

14. Saito K, Miyake K, McNeil PM, Kato K, Yago K, Sugai N: Plasma Membrane Disruption Underlies Injury of the Corneal Endothelium by Ultrasound. Exp Eye Res 1999, 68:43 I-437

15. Rapoport NY, Herron JN, Pitt WG, Pitina L: Micellar Delivery of Doxorubicin and Its Paramagnetic Analog, Ruboxyl to HL-60 Cells: Effect of Micelle Structure on the Intracellular Drug Uptake. / Controlled Release 1999, 58(2): 153-162

16. Marin A, Muniruzzaman M, Rapoport N: Acoustic activation of drug delivery from polymeric micelles: effect of pulsed ultrasound. Journal of Controlled Release 2000, 7 I:239-249

\section{Pre-publication history}

The pre-publication history for this paper can be accessed here:

http://www.biomedcentral.com/1471-2407/2/20/prepub 